\title{
Optimal Quadrature Formulas Using Generalized Inverses. Part I: General Theory and Minimum Variance Formulas*
}

\author{
By C. S. Duris
}

\begin{abstract}
This paper is the first of two papers concerning the derivation of optimal quadrature formulas. In Part I, we develop results concerning generalized inverses and use these results to derive some minimum variance quadrature formulas. The formulas are obtained by inverting appropriate systems of numerical differentiation formulas. The second paper, Part II, will use the same results concerning generalized inverses to derive Sard "best" quadrature formulas.
\end{abstract}

1. Introduction. In an earlier paper [1], a method was described and used to study the truncation error for interpolatory quadrature formulas by inverting systems of differentiation formulas. With the help of generalized inverses (see [6]-[8]), the same idea can be used to derive quadrature formulas without the restriction that the formulas be interpolatory. In this paper, Part I, we will develop some results concerning generalized inverses and use these results to derive some minimum variance formulas as described by Sard in [5]. In a following paper, Part II, the results developed here will be used to formulate a procedure to derive Sard "best" quadrature formulas (see [4]).

We assume that the formulas under consideration have the form

$$
\int_{a}^{b} f(x) d x=\sum_{i=0}^{N} w_{i} f\left(x_{i}\right)+E(f) .
$$

We also assume that (1.1) has $E(f)=0$ for all $f \in \odot_{n}$ where $0 \leqq n \leqq N$. $\odot_{n}$ denotes the set of all polynomials of degree $n$ or less.

Assuming the function $f(x)$ is not known exactly at the points $x_{0}, x_{1}, \cdots, x_{N}$, but only that $\tilde{f}\left(x_{i}\right)$ is known such that $f\left(x_{i}\right)=\tilde{f}\left(x_{i}\right)+\rho_{i}$, we get what we call here the inherent error

$$
I(f)=\sum_{i=0}^{N} w_{i} f\left(x_{i}\right)-\sum_{i=0}^{N} w_{i} \tilde{f}\left(x_{i}\right)=\sum_{i=0}^{N} w_{i} \rho_{i} .
$$

If we ignore computer round-off error, the expression $\sum_{i=0}^{N} w_{i} \tilde{f}\left(x_{i}\right)$ is what is computed to approximate $\int_{a}^{b} f(x) d x$. Thus

$$
\int_{a}^{b} f(x) d x=\sum_{i=0}^{N} w_{i} \tilde{f}\left(x_{i}\right)+T(f)
$$

where $T(f)=I(f)+E(f)$.

Received August 17, 1970.

AMS 1970 subject classifications. Primary 65D30; Secondary 65D25, 65F20.

Key words and phrases. Quadrature formulas, generalized inverses, minimum variance formulas inverting numerical differentiation formulas.

* Prepared under NSF Grant No. GP15405.

Copyright $@ 1971$, American Mathematical Society 
From Krylov [2], we know that if $\left|\rho_{i}\right| \leqq \rho$, a bound on the inherent error $I(f)$ is

$$
|I(f)| \leqq \rho \sum_{i=0}^{N}\left|w_{i}\right|,
$$

and this bound is minimized if the $w_{i}$ 's are all positive. In [3] an analysis is given which indicates that the round-off error itself is likely to be smaller if the weights $w_{i}$ are positive. In [2] and [5] we find that under reasonable statistical assumptions on the $\rho_{i}$ 's, the variance of $I(f)$ is minimized by minimizing $\sum_{i=0}^{N} w_{i}^{2}$.

In this paper, Part I, we develop a procedure for solving

Problem I. Determine the quadrature formula of the form of (1.1) with $E(f)=0$ for $f \in \mathcal{P}_{n}, n<N$, such that $\sum_{i=0}^{N} w_{i}^{2}$ is a minimum.

A computer program has been written employing this procedure and some tables of minimum variance formulas have been computed.

2. Inverting Numerical Differentiation Formulas. We assume $f \in C^{1}[c, d]$ where $[c, d]$ is a finite interval containing the $n+2$ distinct points $z_{0}, \cdots, z_{n+1}$ and the $N+1$ distinct points $x_{0}, \cdots, x_{N}$. The notation $C^{k}[c, d]$ is used to denote the set of functions with $k$ continuous derivatives on $[c, d]$. We permit the possibility that $z_{i}=x_{i}$ for some $i$ and some $j$.

At this point we define

$$
g(x)=\int_{z_{0}}^{x} f(t) d t
$$

which implies that $g \in C^{2}[c, d]$. From the Lagrange interpolation formula, we get

$$
g(x)=l_{0}(x) g\left(z_{0}\right)+l_{1}(x) g\left(z_{1}\right)+\cdots+l_{n+1}(x) g\left(z_{n+1}\right)+R(g ; x)
$$

where

$$
R(g ; x)=\omega_{n+1}(x) g\left[z_{0}, \cdots, z_{n+1}, x\right] .
$$

Here, $g\left[z_{0}, \cdots, z_{n+1}, x\right]$ denotes the $(n+2)$ nd divided difference of $g$ at $z_{0}, \cdots$, $z_{n+1}, x, \omega_{n+1}(x)=\left(x-z_{0}\right)\left(x-z_{1}\right) \cdots\left(x-z_{n+1}\right)$, and

$$
l_{i}(x)=\omega_{n+1}(x) /\left(x-z_{i}\right) \omega_{n+1}^{\prime}\left(z_{i}\right)
$$

for $i=0, \cdots, n+1$.

By differentiating (2.2) and noting that $g^{\prime}(x)=f(x)$, we get

$$
f(x)=l_{1}^{\prime}(x) S_{0,1}+l_{2}^{\prime}(x) S_{0,2}+\cdots+l_{n+1}^{\prime}(x) S_{0, n+1}+R^{\prime}(g ; x)
$$

where

$$
S_{0, i}=\int_{z_{0}}^{z_{i}} f(t) d t
$$

and

$$
R^{\prime}(g ; x)=\omega_{n+1}^{\prime}(x) g\left[z_{0}, \cdots, z_{n+1}, x\right]+\omega_{n+1}(x) g\left[z_{0}, \cdots, z_{n+1}, x, x\right] .
$$

Another representation of (2.5) is

$$
R^{\prime}(g ; x)=\left[\omega_{n+1}^{\prime}(x)-\omega_{n}(x)\right] g\left[z_{0}, \cdots, z_{n+1}, x\right]+\omega_{n}(x) g\left[z_{0}, \cdots, z_{n}, x, x\right]
$$


where $\omega_{n}(x)=\left(x-z_{0}\right) \cdots\left(x-z_{n}\right)$. The form (2.6) is worthwhile when the $(n+2)$ nd divided difference can be related to the $(n+2)$ nd derivative.

The system of equations

$$
\left(\begin{array}{ccc}
l_{1}^{\prime}\left(x_{0}\right) & \cdots & l_{n+1}^{\prime}\left(x_{0}\right) \\
l_{1}^{\prime}\left(x_{1}\right) & \cdots & l_{n+1}^{\prime}\left(x_{1}\right) \\
\vdots & & \vdots \\
l_{1}^{\prime}\left(x_{N}\right) & \cdots & l_{n+1}^{\prime}\left(x_{N}\right)
\end{array}\right)\left(\begin{array}{c}
S_{0,1} \\
\vdots \\
S_{0, n+1}
\end{array}\right)=\left(\begin{array}{c}
f\left(x_{0}\right) \\
f\left(x_{1}\right) \\
\vdots \\
f\left(x_{N}\right)
\end{array}\right)-\left(\begin{array}{c}
R^{\prime}\left(g ; x_{0}\right) \\
R^{\prime}\left(g ; x_{1}\right) \\
\vdots \\
R^{\prime}\left(g ; x_{N}\right)
\end{array}\right)
$$

is obtained by evaluating (2.3) at the points $x_{0}, x_{1}, \cdots, x_{N}$. From Lemma 2.2 of [1], we learn that for $N \geqq n$ the columns of the $(N+1) \times(n+1)$ matrix $L=\left(l_{i i}\right)=$ $\left(l_{i}^{\prime}\left(x_{i}\right)\right)$ are linearly independent. Hence, the rank of $L$ is $n+1$.

We will now indicate how generalized inverses can be used to derive numerical integration formulas from the system of equations (2.7). As done by Rohde in [7], we define the generalized inverses of $L$ to be the set of $(n+1) \times(N+1)$ matrices $B$ such that $L B L=L$. Because $L$ has rank $n+1$ for our case, we may define the generalized inverses of $L$ to be the set

$$
B=\{B \in \operatorname{Tr}(n+1, N+1) \mid B L=I\} .
$$

$\operatorname{Tr}(n+1, N+1)$ denotes the set of all real $(n+1) \times(N+1)$ matrices, and $I$ the $(n+1) \times(n+1)$ identity matrix. The set $B$ is nonempty since $\left(L^{T} L\right)^{-1} L^{T} \in B$.

If we let $B=\left(b_{i j}\right) \in B,(2.7)$ produces the numerical integration formulas

$$
\int_{z_{0}}^{z_{i}} f(t) d t=\sum_{i=0}^{N} b_{i i} f\left(x_{i}\right)+E_{i}(f)
$$

for $i=1,2, \cdots, n+1$. The error term is given by

$$
E_{i}(f)=-\sum_{i=0}^{N} b_{i j} R^{\prime}\left(g ; x_{i}\right)
$$

which can be placed in either of the following two forms

$$
\begin{aligned}
E_{i}(f)=-\sum_{i=0}^{N} b_{i j}\left\{\omega_{n+1}^{\prime}\left(x_{i}\right) g\left[z_{0}, \cdots, z_{n+1}, x_{i}\right]\right. & \\
& \left.+\omega_{n+1}\left(x_{j}\right) g\left[z_{0}, \cdots, z_{n+1}, x_{i}, x_{i}\right]\right\},
\end{aligned}
$$

or

$$
\begin{aligned}
E_{i}(f)=-\sum_{i=0}^{N} b_{i j}\left\{\left[\omega_{n+1}^{\prime}\left(x_{i}\right)-\omega_{n}\left(x_{j}\right)\right] g\left[z_{0}, \cdots, z_{n+1}, x_{i}\right]\right. & \\
& \left.+\omega_{n}\left(x_{i}\right) g\left[z_{0}, \cdots, z_{n}, x_{i}, x_{i}\right]\right\}
\end{aligned}
$$

using (2.5) or (2.6), respectively. Since $g(x)=\int_{x_{0}}^{x} f(t) d t$, we see from either (2.11) or (2.12) that (2.9) has $E_{i}(f)=0$ for $f \in \mathcal{P}_{n}$.

If in (2.12) the terms $b_{i j}\left[\omega_{n+1}^{\prime}\left(x_{i}\right)-\omega_{n}\left(x_{j}\right)\right]$ and $b_{i j} \omega_{n}\left(x_{i}\right)$ all have the same sign (some may be zero) for $j=0,1, \cdots, N$, then

$$
E_{i}(f)=-\left(\sum_{j=0}^{N} b_{i j} \omega_{n+1}^{\prime}\left(x_{i}\right)\right) g\left[z_{0}, \cdots, z_{n}, \bar{x}, \bar{y}\right]
$$


for some $\bar{x}$ and $\bar{y}$ in $[c, d]$. If $g\left[z_{0}, \cdots, z_{n}, \bar{x}, \bar{y}\right]$ is replaced by $(n+2) !^{-1} f^{(n+1)}(\xi)$ for some $\xi \in[c, d]$, then we get

$$
E_{i}(f)=-\left(\frac{1}{(n+2) !} \sum_{j=0}^{N} b_{i j} \omega_{n+1}^{\prime}\left(x_{i}\right)\right) f^{(n+1)}(\xi) .
$$

In any event (even if terms change sign),

$$
\left|E_{i}(f)\right| \leqq C_{i}(n, N) \max _{x \in[c, d]}\left|f^{(n+1)}(x)\right|,
$$

where

$$
C_{i}(n, N)=\frac{1}{(n+2) !} \sum_{i=0}^{N}\left|b_{i j}\right|\left(\left|\omega_{n+1}^{\prime}\left(x_{i}\right)-\omega_{n}\left(x_{i}\right)\right|+\left|\omega_{n}\left(x_{i}\right)\right|\right) .
$$

Note that (2.13) and (2.14) both require that $f \in C^{n+1}[c, d]$.

3. Minimization by Generalized Inverses. Let $\operatorname{Tr}^{*}(n, m)$ designate the set of all $n \times m$ complex matrices. Clearly, $\mathfrak{T}^{*}(n, m)$ is a complex vector space of dimension $n m$ with respect to matrix addition and multiplication by scalars. Let $P=\left(p_{i j}\right)$ be an $m \times m$ positive definite Hermitian matrix. For $A=\left(a_{i j}\right)$ and $B=\left(b_{i j}\right)$ in $\operatorname{Tr}(n, m)$, define

$$
(A, B)=\operatorname{Tr}\left(A P B^{H}\right)=\sum_{i=1}^{n} \sum_{j=1}^{m} \sum_{k=1}^{m} a_{i k} p_{k j} \bar{b}_{i j},
$$

where Tr stands for the trace. It is easy to check that $(A, B)$ is an inner product for $\mathfrak{T}^{*}(n, m)$. Let $L$ be an $m \times n$ matrix. The operator $\mathscr{L}: \mathfrak{T}^{*}(n, m) \rightarrow \mathfrak{T}^{*}(n, n)$ define by $\mathcal{L}(A)=A L$ is a linear operator and if $L$ has rank $n$ the mapping is onto $\mathfrak{T I}^{*}(n, n)$.

THEOREM 3.1. Let $L$ be an $m \times n$ matrix with $m>n$ and rank $L=n$, and let

$$
B=\left\{B \in \operatorname{TI}^{*}(n, m) \mid B L=I\right\} .
$$

Then $W=\left(L^{H} P^{-1} L\right)^{-1} L^{H} P^{-1}$ is the unique matrix in $B$ such that

$$
(W, W)=\min _{B \in \mathbb{B}}(B, B) .
$$

Proof. Since the linear mapping $\mathscr{L}: \mathfrak{T}^{*}(n, m) \rightarrow \mathfrak{T}^{*}(n, n)$ defined by $\mathscr{L}(A)=A L$ is onto, the dimension of $\operatorname{ker}(\mathscr{L})=\left\{A \in \mathfrak{T}^{*}(n, m) \mid A L=0\right\}$ is $p=n(m-n)$. Let $A_{1}, A_{2}, \cdots, A_{p}$ be an orthogonal basis for $\operatorname{ker}(\mathscr{L})$. The set $@$ can now be represented by

$$
B=\left\{B \mid B=W+\sum_{i=1}^{p} \alpha_{i} A_{i}\right\}
$$

where the $\alpha_{i}$ 's are arbitrary complex numbers. Now consider $B \in B$

$$
\begin{aligned}
(B, B) & =\left(W+\sum_{i=1}^{p} \alpha_{i} A_{i}, W+\sum_{i=1}^{p} \alpha_{i} A_{i}\right) \\
& =(W, W)+\sum_{i=1}^{p}\left[\alpha_{i}\left(A_{i}, W\right)+\bar{\alpha}_{i}\left(W, A_{i}\right)\right]+\sum_{i=1}^{p}\left|\alpha_{i}\right|^{2}\left(A_{i}, A_{i}\right) .
\end{aligned}
$$

Since

$$
\left(A_{i}, W\right)=\operatorname{Tr}\left(A_{i} P P^{-1} L\left(L^{H} P^{-1} L\right)^{-1}\right)=0,
$$


we get

$$
(B, B)=(W, W)+\sum_{i=1}^{p}\left|\alpha_{i}\right|^{2}\left(A_{i}, A_{i}\right)
$$

Clearly, $(B, B)$ is a minimum if and only if $\alpha_{i}=0$ for all $i$. This proves Theorem 3.1.

Let $w_{(i)}=\left(w_{i 1}, w_{i 2}, \cdots, w_{i m}\right)$ be the $i$ th row of $W$ defined in Theorem 3.1. The following corollary is useful as a minimization procedure for integration formulas.

COROLlaRY 3.2. Let $\mathrm{B}_{i}=\left\{b \in C^{m} \mid b^{H} L=e_{i}\right\}$ where $e_{i}$ is the row vector of all zeros except for $a 1$ in the ith position. Then $w_{(i)}^{H} \in B_{i}$ is the unique vector such that

$$
\boldsymbol{w}_{(i)} P \boldsymbol{w}_{(i)}^{H}=\sum_{j=1}^{m} \sum_{k=1}^{m} w_{i k} p_{k j} \bar{w}_{i j}=\min _{b \in B_{i}} b^{H} P b .
$$

Proof. Assume there exists a $b \in \bigotimes_{i}$ such that $b^{H} P b \leqq w_{(i)} P w_{(i)}^{H}$. Then the matrix $V$ formed from $W$ by replacing $w_{(i)}$ with $b^{H}$ would still have $V L=I$. If $b^{H} \neq w_{(i)}$, we would have $(V, V) \leqq(W, W)$ and $V \neq W$. This contradicts Theorem 3.1, thus proving the desired corollary.

4. Minimum Variance Integration Formulas. In the introduction, we stated

Problem I. Determine the quadrature formulas of the form

$$
\int_{a}^{b} f(x) d x=\sum_{i=0}^{N} w_{i} f\left(x_{i}\right)+E(f)
$$

with $E(f)=0$ for $f \in \mathcal{P}_{n}, 0 \leqq n \leqq N$, such that $\sum_{i=0}^{N} w_{i}^{2}$ is a minimum.

As mentioned in the introduction, formulas for which $\sum_{i=0}^{N} w_{i}^{2}$ is a minimum minimize the variance of the inherent error. Using Corollary 3.2 and Eqs. (2.7) and (2.9), we get

THEOREM 4.1. The vector $w^{T}=\left(w_{0}, \cdots, w_{N}\right)$ solving Problem I is the $(n+1)$ st row in the $(n+1) \times(N+1)$ matrix $\left(L^{T} L\right)^{-1} L^{T}$ where $L=\left(l_{i j}\right)$ with

$$
l_{i j}=l_{j}^{\prime}\left(x_{i}\right)=\left.\frac{d}{d x}\left(\frac{\omega_{n+1}(x)}{\left(x-z_{i}\right) \omega_{n+1}^{\prime}\left(z_{j}\right)}\right)\right|_{x=x_{i}} .
$$

Here $\omega_{n+1}(x)=\left(x-z_{0}\right)\left(x-z_{1}\right) \cdots\left(x-z_{n+1}\right)$, where $z_{0}=a$ and $z_{n+1}=b$. (The $n$ other points $z_{1}, \cdots, z_{n}$ can be arbitrary.)

The proof of this theorem is an immediate consequence of Eq. (2.9) and Corollary 3.2 with $P=I$ and $L$ the matrix of the system of equations in (2.7).

A FORTRAN program has been written to solve Problem I given $n, N, a, b$, and $x_{0}, x_{1}, \cdots, x_{N}$. The program also requires $z_{1}, z_{2}, \cdots, z_{n}, z_{0}=a$ and $z_{n+1}=b$ for defining $\omega_{n+1}(x)=\left(x-z_{0}\right)\left(x-z_{1}\right) \cdots\left(x-z_{n+1}\right)$. The points $z_{1}, \cdots, z_{n}$ can be arbitrary distinct numbers not equal to $a$ or $b$. The program computes the $(n+1)$ st row in $W=\left(L^{T} L\right)^{-1} L$ using the elementary unitary Hermitian matrices of Householder (see Hanson and Lawson [9]).

Table I gives the minimum variance weights $w_{0}, w_{1}, \cdots, w_{N}$ for the formula

$$
\int_{0}^{N} f(x) d x=\sum_{i=0}^{N} w_{i} f(i)+E(f)
$$

having $E(f)=0$ for $f \in \mathcal{P}_{n}$, i.e. having algebraic degree of precision $n$. For formulas of the form of (4.3), we get $w_{i}=w_{N-i}$. Thus the table only lists $w_{i}$ for $i=$ 
TABLE I

$\begin{array}{cc}N & 2 \\ W(0) & .333333333333 \\ W(1) & 1.333333333333 \\ W(2) & \\ C & .111111111111 \\ \text { VAR } & .500000000000\end{array}$

$N$
$W(0)$$\quad .500000000000$

W(1) $\quad .857142457143$

W(2) $\quad 1.07142857142 .9$

W(3) 1.142857142857

if 4 )

C

2.1482.14285714

.154761904762

$\begin{array}{cc}N & 4 \\ W(0) & .311111111111 \\ W(1) & 1.42222222222 ? \\ W(2) & .53333333333 \\ W(3) & \\ C & .008465608466 \\ \text { VAR } & .282716049383\end{array}$

$\begin{array}{ll}N & N \\ W(0) & .384149184149\end{array}$

W(1) 1.080341880342

$w(2) \quad 1.123853923854$

$W(3) \quad .968453764454$

W(4) $\quad .8864021486402$

C 1.351446212681

VAR $\quad .172141068808$
ALGF.BRAIC DEGREF OF PRECISINN 3 3

3
.375000000000
.112500000000
.375000000000
.312500000000
7
.534722222227
.826388888889
1.02083333333
1.118055555556
4.738975694444
.133101851857

5

.461309523810 .907738095238

1.130952380952

.896654885913

.185267857 .143

. 306031746032

.231746031746

8

.565656565657

.808080808081

.981240981241

1.085137085137

1.119769119769

8.591822991873

.116883116883
9
.325360733017
1.312430226024
.876187874625
.868247689810
1.117773476523
.361037620649
.113563197336
AL.GFBRAIC DFGRFF OF PRECISINN 5 5

.329851111111

$1.3020 R 3333333$

.868055555556

.043300182048

.204619984568

9

.402010489510

1.031341118881

1.131687062937

1.02.1547202797

.913374125874

2.961937593656

.108244099650
.34805194805 ? 1.211688311688 1.020779220779 .838961038961 .1825788497?2 .165735930736
7

. 366130050505 1.139488636364 1.0930871 ? 1212

.901294191919

.547389230092

.140394132295

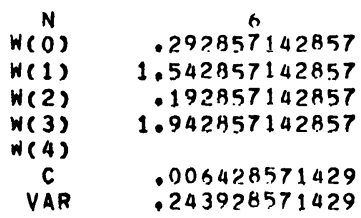
ALGFBRAIC DEGREF OF PRECISIIIN 7
. 30422 ? 4537037
1.449016203704
.535937500000
1.2.10821759259
032011796898 .161041596291
.3149877283?
$1.374 ? 78067611$
.743465916799
.951163404497
1. 232209765543
.119802533348
.131390041274

9

.593181818182

.797727272727

.951136363636

1.053409090909

1.104545454545

15.660383522727

.104261363636

$0,1, \cdots, N_{m}$, where $N_{m}=N / 2$ or $(N-1) / 2$. The constant $C(n, N)$ giving the bound

$$
|E(f)| \leqq C(n, N) \max _{x \in[0, N]}\left|f^{(n+1)}(x)\right|
$$

is computed by (2.15). This $C(n, N)$ is denoted by $C$ in the table. The parameter

$$
\sigma^{2}(n, N)=\left(\sum_{i=0}^{N} w_{i}^{2}\right) / N^{2}
$$

is given in Table I and is denoted there by VAR. This VAR parameter supplies a comparison of the variance when evaluating $\int_{0}^{1} f(x) d x$ approximately using (4.3). The results were computed to 15 decimal digits, but only 12 digits are given in the tables. Table I, for algebraic degree of precision $n=3$, agrees with the results given 
by Sard in [5]. For example, for $N=6$ with degree of precision 3, we have

$$
\begin{aligned}
\int_{0}^{6} f(x) d x= & \frac{1}{2} f(0)+\frac{6}{7} f(1)+\frac{15}{14} f(2)+\frac{8}{7} f(3) \\
& +\frac{15}{14} f(4)+\frac{6}{7} f(5)+\frac{1}{2} f(6)+E(f)
\end{aligned}
$$

with

$$
|E(f)|<2.1482 \max \left|f^{(4)}(x)\right| \text { and } \sigma^{2}(3,6)=0.154761904762
$$

$\begin{array}{cc}N & 3 \\ W(0) & -.3750001100000 \\ W(1) & 1.541666666667 \\ W(2) & -2.458333333333 \\ W(3) & 2.291666666666 \\ W(4) & \\ W(5) & \\ W(6) & \\ C & \\ \text { VAR } & 1.01453993055 \\ & \end{array}$

$\begin{array}{cc}N & 7 \\ w(0) & -.251893939394 \\ w(1) & .304924742424 \\ w(2) & .283639971140 \\ w(3) & -.0329181770418 \\ w(4) & -.361922799423 \\ w(5) & -.470544733045 \\ w(6) & .074044) 11544 \\ w(7) & 1.404671717172 \\ w(8) & \\ w(9) & \\ C & 7.776189630682 \\ \text { VAR } & 2.524395743146\end{array}$

TARLE II

ALGFBRAIC OFGREF OF PRECISION 3

$$
4
$$

$-.326785714286$ .93214285714 .3

.0410047619048

$-1.151190476190$

1.96488095 ? 341

1.701468253968 6. 337276785714

8

$-.236111111111$ .2203?R282828 .271194083694 . OR1259018750

. .184704184704

$-.361922799423$

$-.285624098124$

. 008964646465 1.286616161616

12.736264430014 2.130174512987 3.132722594246
$-.222552447552$ .159537684538 .245056332556 .136956099456

-.061810411810

.248290598201

$-.319531857032$

-.17258158508 ? .295512820513 1.187703962704 19.371175962 .348 1.849113733489 6

$-.270833333333$ .427579365079 .254960317460 $-.253968253968$ . .564484126984 $-.141865079365$ 1.548611111111 5.212768518519 3.122643849206

ALGFRRAIC, DEGRFF OF PRECISION 5

$$
6
$$

$-.295747655123$

1.444624819625

$-7.438298160173$

.8468975146898

$? .495739617605$

$-3.727597402597$

2.6743912 .33766

1.789155158934

$3.2592 \times 5278819$ 3.259245278819
36.112958109117

$$
\begin{array}{r}
-.270092754468 \\
1.093300796426 \\
-1.217718045843 \\
-.509064199689 \\
1.475667735043 \\
.643665015540 \\
-2.608641705517 \\
2.442883158508 \\
5.230551707291 \\
18.279763142914
\end{array}
$$

\section{8}

$-.250145687646$ .855562839938 $-.595182595183$ $-.717426670552$ .428982128982 1.050449203574

-.169308469308 $-1.858204642580$ $2.255 ? 73892774$ 8.407463013524 11.518 ค2 3366544 

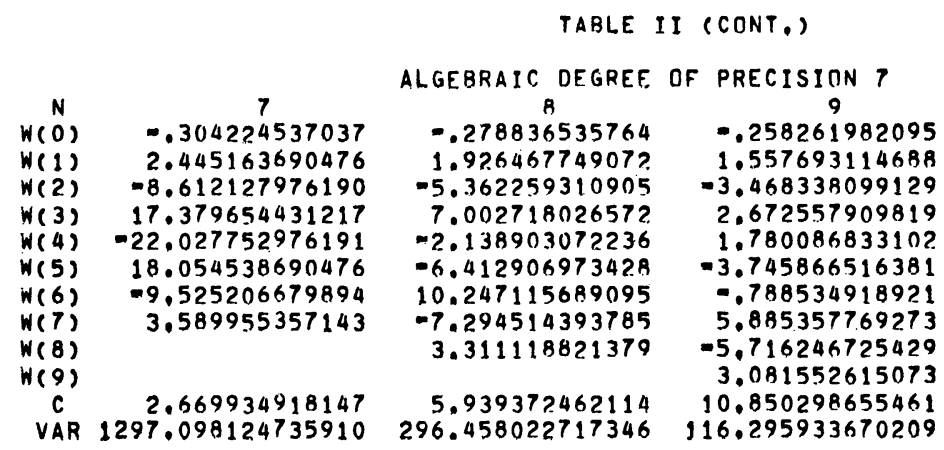

Table II gives the minimum variance weights $w_{0}, \cdots, w_{N}$ for the Adams-Bashforth type predictor formulas, and Table III gives the minimum variance weights for the Adams-Moulton type corrector formulas. The symmetry occurring in Table I does not occur in Tables II or III. The $C$ again denotes $C(n, N)$ computed by formula (2.15), and VAR here denotes

$$
\sigma^{2}(n, N)=\sum_{i=0}^{N} w_{i}^{2} .
$$

No division by $N^{2}$ is made since the integral is already over the unit interval $[N, N+1]$ or else the unit interval $[N-1, N]$. As an example, consider the case $N=6$ with algebraic degree of precision 5. From Table II, the predictor formula is

$$
\begin{aligned}
& \int_{6}^{7} f(x) d x=-0.295747655123 f(0)+1.444624819625 f(1) \\
&-2.438298160173 f(2)+0.846897546898 f(3) \\
&+2.495729617605 f(4)-3.727597402597 f(5) \\
&+2.674391233766 f(6)+E(f) \\
& \text { TABLE III }
\end{aligned}
$$

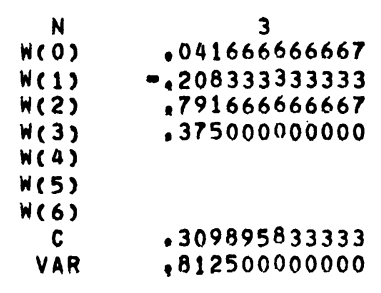

7

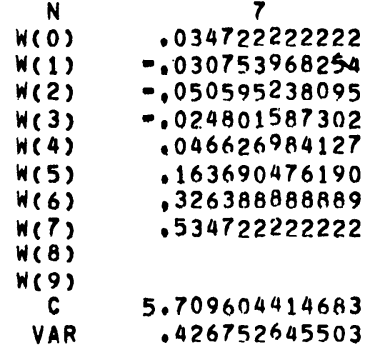

ALGEBRAIC DEGREE. OF PRECISION 3 4 .060119047619 $-.198809523810$ .152380952381 .551190476190 .435119047619

.857698412698 .559499007937

8

.020622895623

.006523569024

. .027807840308

.032918470418

$-.011544011544$ .046626984927 .151905964406 .314604377104 .545033670034

$8.68837882796 ?$ .423745139891
.007634032634 .007051282051 . .00737179487 ? $-.023203185703$

$-.028010878011$

.009362859363 .045172882673 .148028360528 . 311635586636 .548426573427

12.845799319315 . 423414432789
6

.048611111111

.0070436507937

. .064484126984 .031744603175 - 183531746032 .356150793651 .514880952381 3,462880291005 .438120039683 


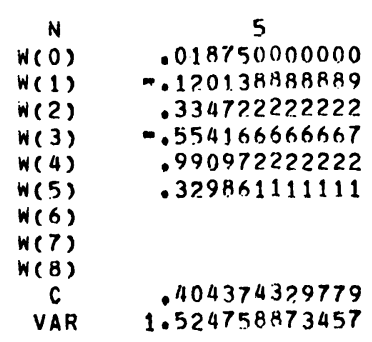

$\begin{array}{cc}N & 9 \\ W(0) & .035763403263 \\ W(1) & .0109380341880 \\ W(2) & .048112859363 \\ W(3) & .104561965812 \\ W(4) & .0001181492618 \\ W(5) & -.128925796426 \\ W(6) & -.097831196581 \\ W(7) & .178897630148 \\ W(8) & .539819347319 \\ W(9) & .428863636364 \\ C & 9.192981616763 \\ \text { VAR } & .560017258590\end{array}$

TAPLE III (CONT.)

ALGEBRAIC DEGREF. DF PRECISION 5 6

.024391233766

. .127597402597

.245779617605

$-.15310245310 ?$

$-.188298160173$

. R44624819625

.354252344877

1.009714105339

.975041442450
.029766414141

-.128693181818

.165814393939

.033112373737

- .216887626263

.015814393939

.721306818182

.379766414141

\subsection{8} .757835209736
8

0.33673271173

$-.121763306138$

.098601398601

.098467851593

$-.093162393162$

. .166377719503

.125252525253

.620287004662

.405021367521

4.842623076417

.636225593947

with

$$
|E(f)| \leqq 3.2593 \max \left|f^{(6)}(x)\right| \text { and } \sigma^{2}(5,6)=36.112958109117 .
$$

Note that in Table III for $N=6$ with degree of precision 5 , the corresponding corrector formula has the smaller error bound

$$
|E(f)| \leqq 1.0097 \max \left|f^{(6)}(x)\right|
$$

as well as the smaller variance

$$
\sigma^{2}(5,6)=0.975041442450 \text {. }
$$

As can be seen by comparing Tables II and Tables III, this holds true in all cases considered. Hence, the corrector formulas would appear to give considerable improvement over the predictor formulas. 
Acknowledgment. Thanks are due to Miss Alice Stanley for carrying out the FORTRAN programming needed.

Department of Mathematics

Drexel University

Philadelphia, Pennsylvania 19104

1. C. S. DuRIS, "A simplex sufficiency condition for quadrature formulas," Math. Comp., v. 20,1966 , pp. 68-78. MR 33 \#5118.

2. V. I. KRYLOV, Approximate Calculation of Integrals, Fizmatgiz, Moscow, 1959; English transl., Macmillan, New York, 1962. MR 22 \#2002; MR 26 \#2008.

3. P. J. Davis \& P. Rabinowitz, Numerical Integration, Blaisdell, Waltham, Mass., 1967. MR 35 \#2482.

4. A. SARD, Linear Approximation, Math. Surveys, no. 9, Amer. Math. Soc., Providence, R.I., 1963. MR 28 \# 1429.

5. A. SARD, "Smoothest approximation formulas," Ann. Math. Statist., v. 20, 1949, pp. 612-615. MR 12, 84.

6. T. N. E. Greville, "Some applications of the pseudo-inverse of a matrix," SIAM Rev., v. 2, 1960, pp. 15-22. MR 22 \# 1067.

7. C. A. ROHDE, "Some results on generalized inverses," SIAM Rev., v. 8, 1966, pp. 201-205. MR 35 \#1608.

8. R. P. TEWARSON, "On some representations of generalized inverses," SIAM Rev., v. 11, 1969, pp. 272-276. MR 39 \#6890.

9. R. J. Hanson \& C. L. Lawson, "Extensions and applications of the Householder algorithm for solving linear least squares problems," Math. Comp., v. 23, 1969, pp. 787-812. 